Supporting Information for

\title{
Optoelectronic Property Modulation in Chiral Organic Semiconductor/Polymer Blends
}

Inho Song,,$^{\dagger, \perp}$,Jaeyong Ahn, $,{ }^{\dagger}, \perp$ Xiaobo Shang, ${ }^{\dagger, \dagger}$ and Joon Hak Oh*,

${ }^{\dagger}$ School of Chemical and Biological Engineering, Institute of Chemical Processes, Seoul National University, 1 Gwanak-ro, Gwanak-gu, Seoul 08826, Republic of Korea

${ }^{\ddagger}$ Chemistry Research Laboratory, Department of Chemistry, University of Oxford, Mansfield Road, Oxford OX1 3TA, UK

*E-mail: joonhoh@snu.ac.kr 

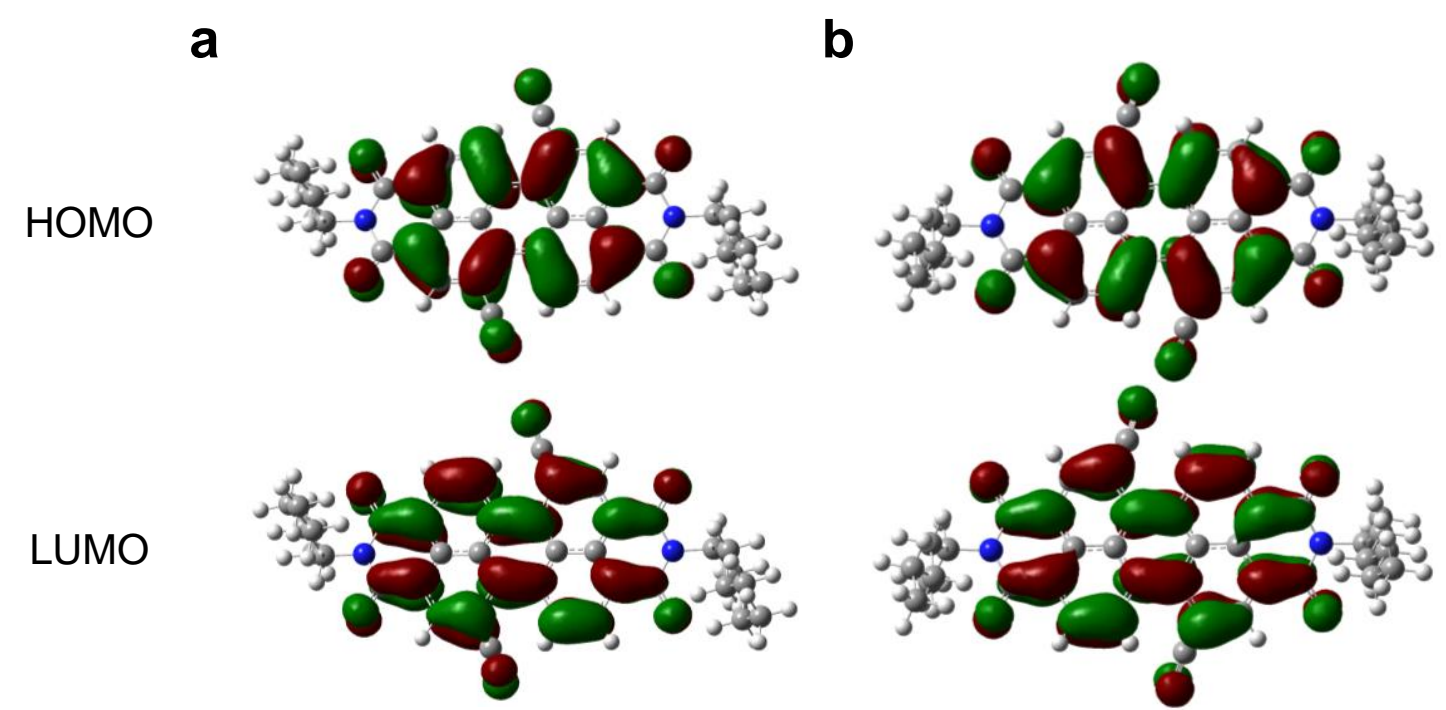

Figure S1. HOMO and LUMO electron distributions of a) (R)-CPDI-CN2-C6 and b) $(S)$ CPDI-CN2-C6 obtained by DFT calculations. 
a

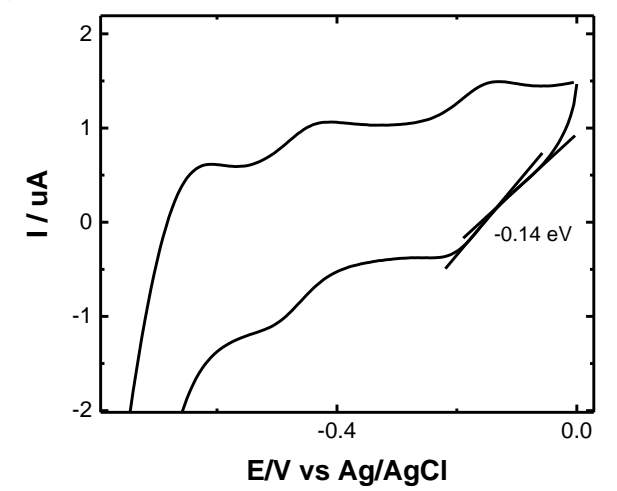

b

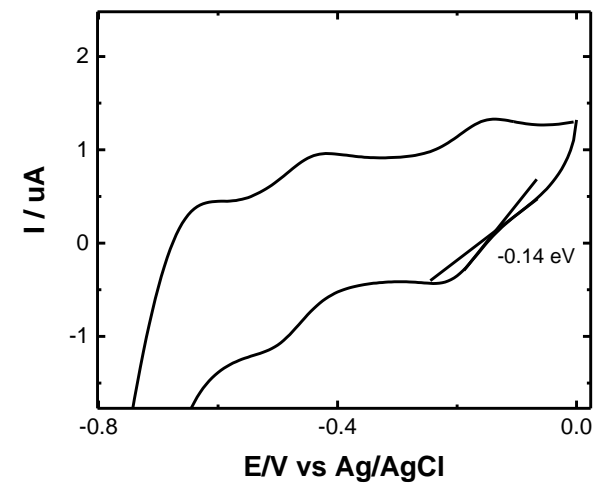

Figure S2. Cyclic voltammetry results of a) (S)-CPDI-CN2-C6 and b) (R)-CPDI-CN2-C6 solution $\left(1.0 \times 10^{-4} \mathrm{M}\right.$ in chloroform $)$. 


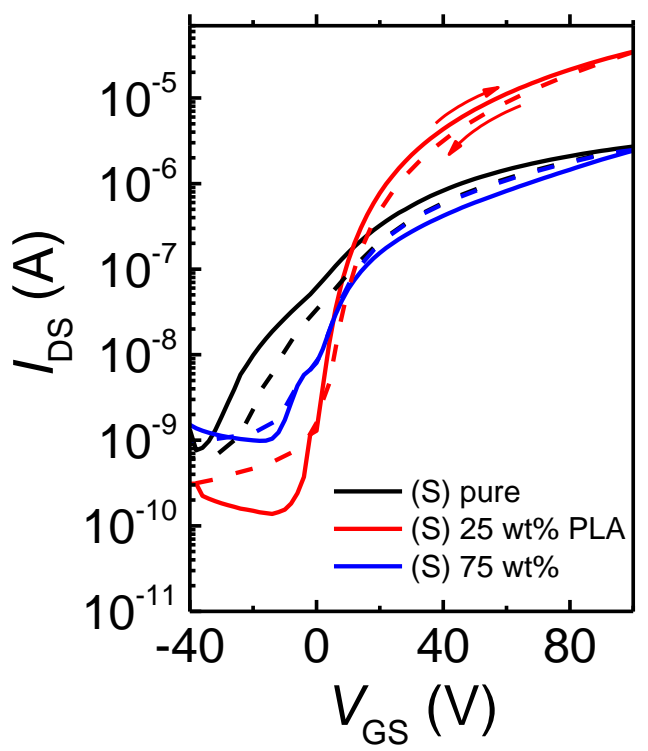

Figure S3. Hysteresis behaviors of PLA-blended CPDI-CN2-C6 thin films with different compositions of PLA in the dark condition. 


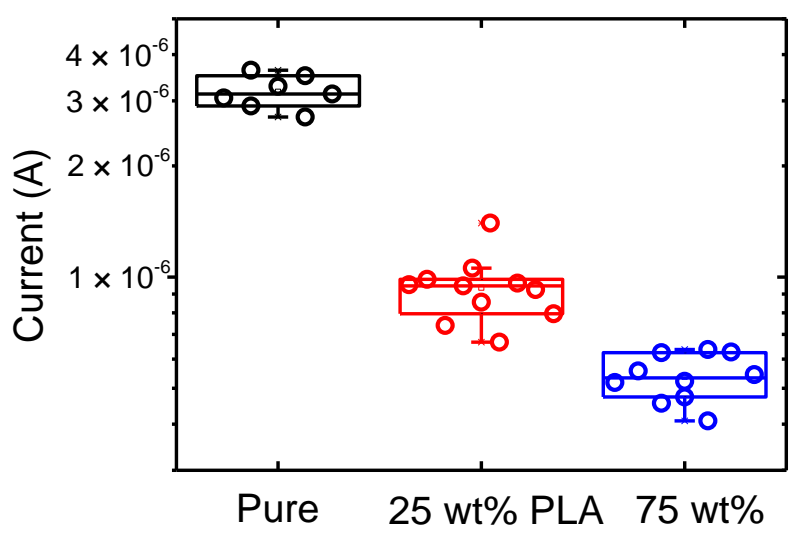

Figure S4. Drain current distributions of several devices depending on PLA composition ( $V_{\mathrm{GS}}$ $\left.=100 \mathrm{~V}, V_{\mathrm{DS}}=100 \mathrm{~V}\right)$. 


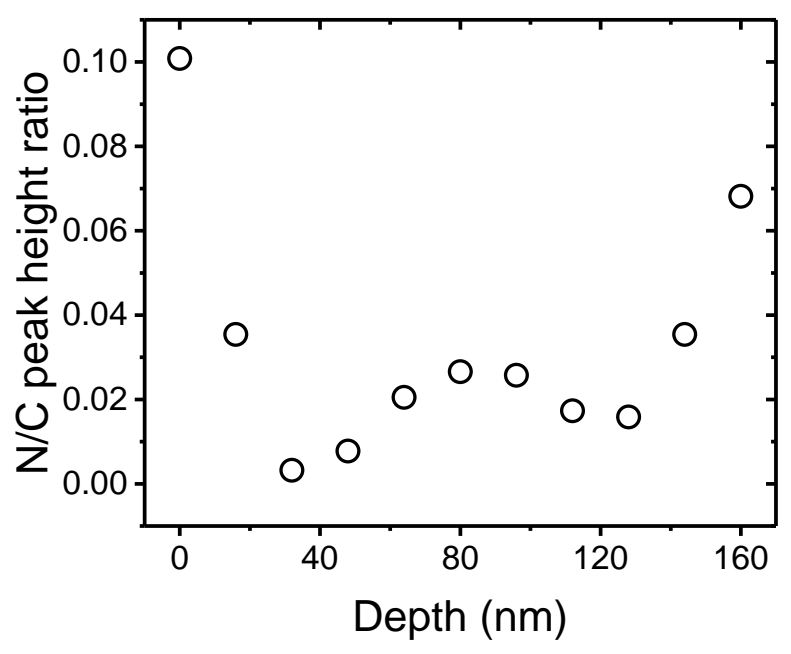

Figure S5. XPS characterization of nitrogen/carbon peak height ratios, showing the vertical distribution of $75 \mathrm{wt} \%$ PLA-blended film. 


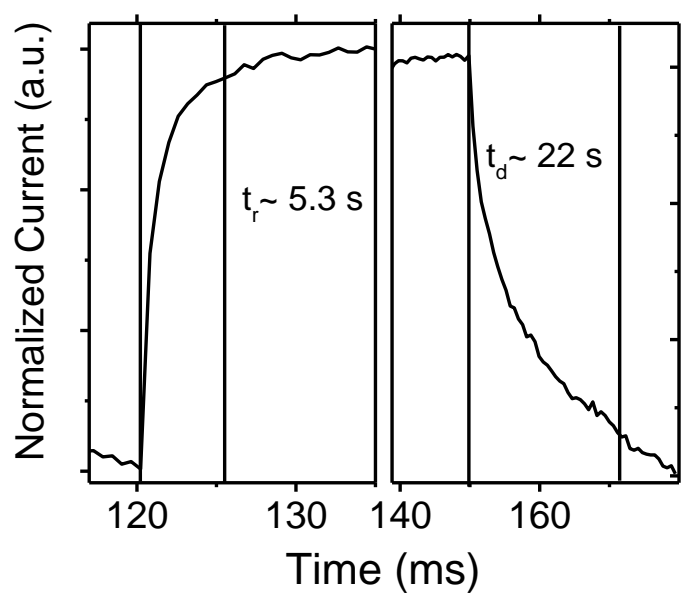

Figure S6. Rise/decay time of $(R a c)$-CPDI-CN2-C6 thin film based OPTs $\left(V_{\mathrm{GS}}=40 \mathrm{~V}, V_{\mathrm{DS}}=\right.$ $100 \mathrm{~V})$. 
a

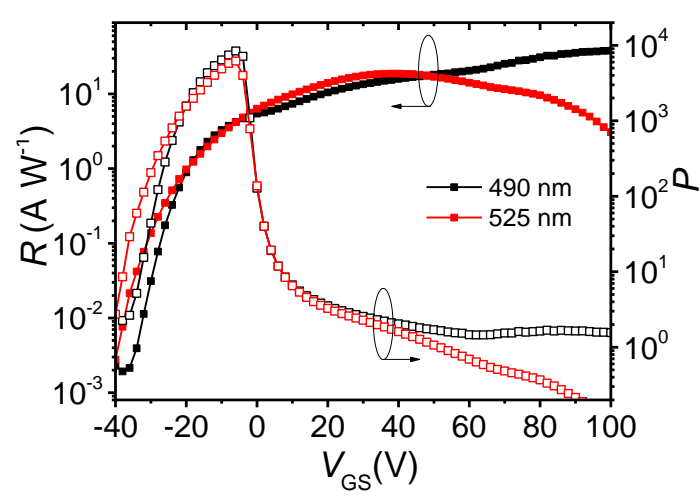

b

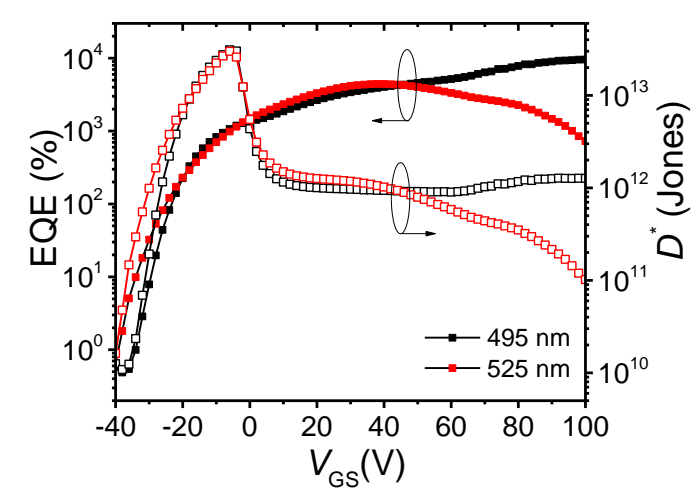

Figure S7. a) $R$ and $P$, b) EQE and $D^{*}$ of $75 \mathrm{wt} \%$ PLA-blended (Rac)-CPDI-CN2-C6 thin films under 490 and $525 \mathrm{~nm}$ light illumination. 


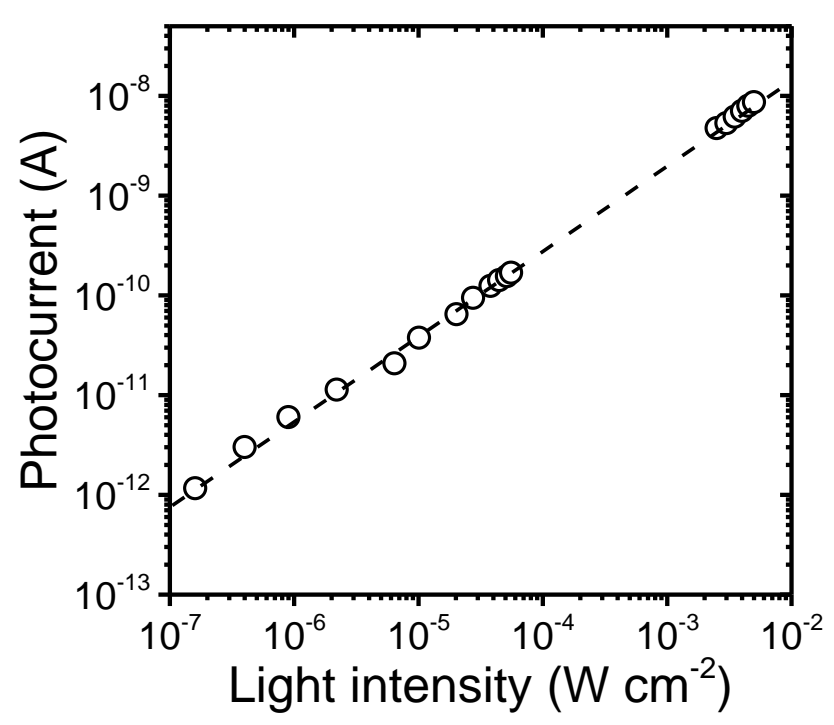

Figure S8. Photocurrent of (Rac)-CPDI-CN2-C6 with $75 \mathrm{wt} \%$ PLA thin film based OPTs measured at $-20 \mathrm{~V}$ as a function of incident light irradiance $(\lambda=532 \mathrm{~nm})$. The irradiance was ranged from $160 \mathrm{nW} \mathrm{cm} \mathrm{cm}^{-2}$ to $5 \mathrm{~mW} \mathrm{~cm} \mathrm{~cm}^{-2}$, which was the lower and upper limit of our illumination facilities, respectively. 
a

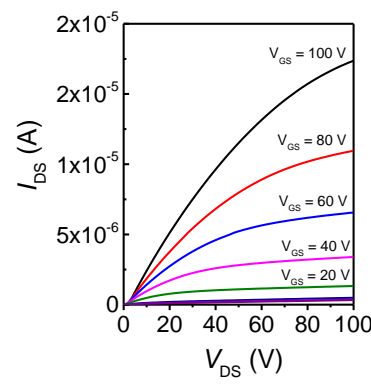

e

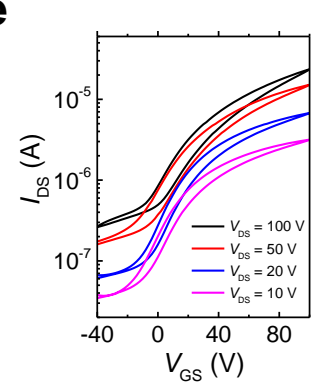

b

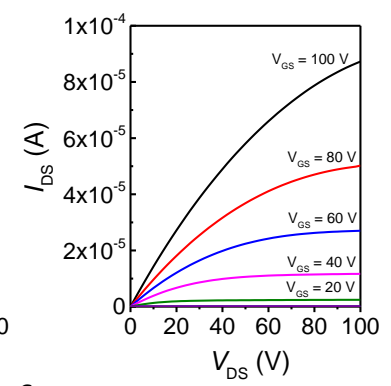

f

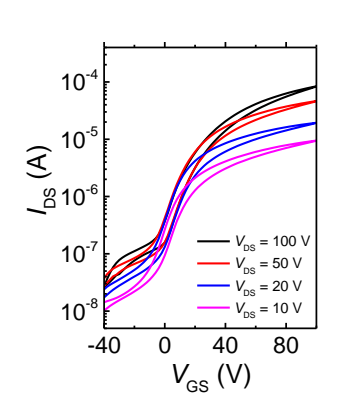

C

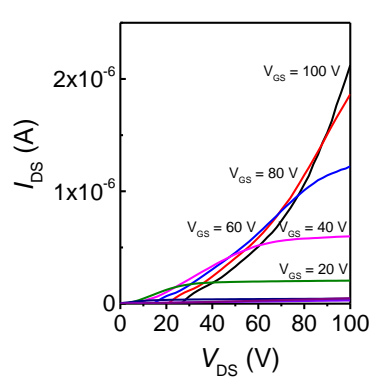

g

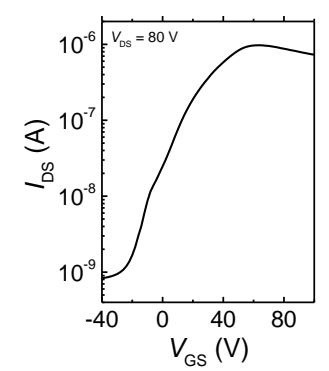

d

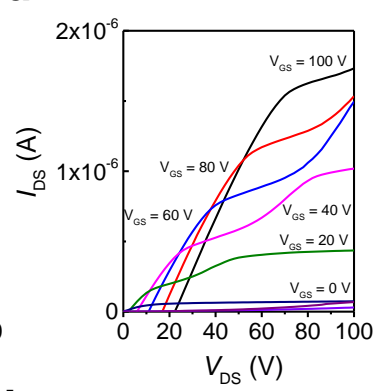

h

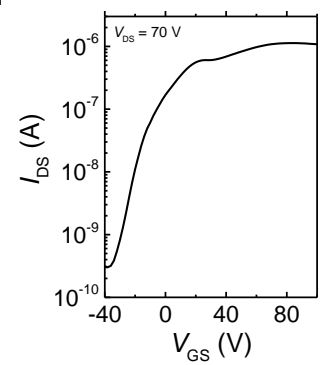

Figure S9. Output characteristics of a) pure, b) $25 \mathrm{wt} \%$ PLA-blended, c) $75 \mathrm{wt} \%$ PLA-blended (S)-CPDI-CN2-C6, and d) 75 wt\% PLA-blended (Rac)-CPDI-CN2-C6. Transfer characteristics of e) pure, f) $25 \mathrm{wt} \%$ PLA-blended, g) $75 \mathrm{wt} \%$ PLA-blended (S)-CPDI-CN2-C6, and h) $75 \mathrm{wt} \%$ PLA-blended (Rac)-CPDI-CN2-C6 under linear regime. 


\section{$500 \mathrm{~nm}$}

Figure S10. AFM images of $25 \mathrm{wt} \%$ PLA-blended (S)-CPDI-CN2-C6 film. 
Table S1. Energy levels of enantiomeric CPDI-CN2-C6 from CV measurement and DFT calculation.

\begin{tabular}{cccc}
\hline & Material & Orbital & Energy level (eV) \\
\hline \multirow{2}{*}{ DFT } & $(R)$-CPDI-CN2-C6 & LUMO & -4.14 \\
& & HOMO & -6.60 \\
& $(S)$-CPDI-CN2-C6 & LUMO & -4.14 \\
& & HOMO & -6.61 \\
\hline \multirow{2}{*}{ CV } & $(R)$-CPDI-CN2-C6 & LUMO & -4.23 \\
& & HOMO & -6.52 \\
& $(S)-C P D I-C N 2-C 6$ & LUMO & -4.23 \\
& & HOMO & -6.52 \\
\hline
\end{tabular}

